Mark K. Wax, MD, Section Editor

\title{
INFRAHYOID FASCIO-MYOCUTANEOUS FLAP AS AN ALTERNATIVE TO FREE RADIAL FOREARM FLAP IN HEAD AND NECK RECONSTRUCTION
}

\author{
Alberto Deganello, MD, ${ }^{1}$ Valentina Manciocco, MD, ${ }^{1}$ Gilles Dolivet, MD, PhD, ${ }^{2}$ \\ C. René Leemans, MD, PhD, ${ }^{3}$ Giuseppe Spriano, MD $^{1}$ \\ ${ }^{1}$ Department of Otolaryngology/Head and Neck Surgery, National Cancer Institute "Regina Elena," \\ Rome, Italy. E-mail: adeganello@hotmail.com \\ ${ }^{2}$ Head and Neck Surgery Unit, Centre "Alexis Vautrin," Nancy, France \\ ${ }^{3}$ Department of Otolaryngology/Head and Neck Surgery, VU Medical Center, Amsterdam, The Netherlands
}

Accepted 1 June 2006

Published online 17 January 2007 in Wiley InterScience (www.interscience.wiley.com). DOI: 10.1002/hed.20512

\begin{abstract}
Background. The use of microvascular free flaps is currently the favored method for the reconstruction of defects after resection of head and neck cancer. The flap most commonly used for head and neck reconstruction is the free radial forearm flap, but the less popular infrahyoid flap represents a good alternative in selected cases. This flap has proven to be helpful in the reconstruction of a wide range of moderate-sized head and neck defects.

Methods. We reviewed a series of 13 patients with defects resulting from cancer of the head and neck, who underwent infrahyoid flap reconstruction as an alternative to free radial forearm flap. The series includes 12 squamous cell carcinomas arising from the oral cavity and oropharynx, and 1 Merkel cell carcinoma of the submental skin. In the harvesting of the flap, the technical modifications recently suggested by Dolivet et al were used in all cases. Furthermore, another technical change has been introduced so creating a new infrahyoid facio-myocutaneous flap (IHFMCF).

The surgical technique is described in detail.

Results. No total or partial flap necrosis was experienced. All reconstructions healed quickly without wound complications and with good functional results. The healing process in the donor site was excellent in every case with good aesthetic results.

Conclusions. The IHFMCF is a versatile, reliable, and convenient flap suitable for repairing small and medium-sized
\end{abstract}

Correspondence to: A. Deganello

๑ 2007 Wiley Periodicals, Inc. defects of the oral cavity and oropharynx and obviates the need for a microvascular reconstruction. (C2007 Wiley Periodicals, Inc. Head Neck 29: 285-291, 2007

Keywords: head and neck reconstruction; infrahyoid flap; free radial forearm flap

The main goals of modern head and neck reconstructive surgery are adequate wound healing, restoration of function, and appearance. In the preoperative planning, it is crucial to determine which reconstructive procedure will be most suitable for the patient to optimize functional outcome after cancer ablation. As a general rule, an optimal reconstruction should enhance the residual function allowing good motility of the preserved structures around the resected area, guarantee a quick and safe healing process, and provide a restoration of form with acceptable aesthetic results. The application of microvascular free flaps is the most widespread method currently employed for the reconstruction of extensive defects after resection of head and neck cancer because of their versatility and reliability. The 
flap most commonly used for head and neck reconstruction is the free radial forearm flap (FRFF). ${ }^{1}$

The realization that not all patients are suitable for a free flap reconstruction and that not every defect strictly requires a free flap transfer to achieve a good functional result raises the necessity to find alternatives. The pectoralis major flap and temporalis flap are the most used pedicled flaps in head and neck reconstruction, but the less popular infrahyoid myocutaneous flap (IHMCF) represents an interesting alternative in selected cases.

In 1980, Wang and Shen ${ }^{2}$ first described the IHMCF for head and neck reconstruction. In spite of its limited rotation arch, this flap has proven to be helpful in the reconstruction of a wide range of moderate-sized head and neck defects (intraoral, pharyngeal, and parotid region) ${ }^{3-8}$ The major blood supply of the IHMCF is derived from the superior thyroid artery, which is the first branch of the external carotid artery. The higher the bifurcation of the common carotid artery, the more convenient it is to transfer the IHMCF upward. All the branches of the superior thyroid artery, except its posterior branch to the thyroid gland, have tiny tributaries entering the infrahyoid muscles and the overlying cervical skin. To increase success rate, Wang in 1991 recommended including the sternal edge of the sternocleidomastoid (SCM) muscle to protect the platysma and the SCM branches of the superior thyroid artery. ${ }^{9}$

The complication rate reported in the literature is extremely variable, ranging from $3 \%$ to $47 \%{ }^{3-7}$; the main problems are related to the reliability of the skin paddle for insufficient venous drainage. Recently, Dolivet et $\mathrm{al}^{10}$ described a modification of the original surgical technique to improve drainage ${ }^{2-5}$ : the detachment of the infrahyoid muscles from the hyoid bone is carried out in a subperiosteal plane to preserve microvenous drainage toward digastric triangle network. They also changed the cervical incision from an inverted $\mathrm{T}$ to an inverted $\mathrm{Z}$, with better aesthetic results. In this article, a series of 13 infrahyoid flap reconstructions for selected tumors of the oral cavity, pharynx, and cervical skin is presented as an alternative to FRFF reconstruction. All patients underwent reconstruction using the improvements of Dolivet et $\mathrm{al}^{10}$ with the addition of a personal surgical modification.

\section{MATERIALS AND METHODS}

From October 2003 to April 2005, 13 patients, 11 men and 2 women, underwent infrahyoid flap reconstruction after cancer ablation and neck dissection, in a single-stage procedure. The ages of the patients ranged from 29 to 81 years, with median age of 60 years. The series included 12 squamous cell carcinomas (SCC) arising from the mucosa of the oral cavity and oropharynx, and 1 Merkel cell carcinoma (MCC) of the submental skin. Disease was staged according to the 6th edition of the TNM classification established by the UICC/AJCC. ${ }^{11}$ All the reconstructions were performed by the first author.

In this series, infrahyoid flap reconstruction was chosen as an alternative to FRFF reconstruction. Selection criteria were a defect estimated as small or medium size or the presence of relative general contraindications to a microvascular free flap reconstruction. In the harvest of the flap, the technical modifications suggested by Dolivet et $\mathrm{al}^{10}$ were used in all cases with an extra technical change. In this series, to increase venous drainage toward the median cervical fascia, a portion of the superficial cervical fascia is included in the flap creating actually a new infrahyoid fasciomyocutaneous flap (IHFMCF).

The dimensions of the skin paddle ranged from a minimum of $5 \mathrm{~cm}$ in length and $3 \mathrm{~cm}$ in width for a pharyngolaryngeal reconstruction to a maximum of $9 \mathrm{~cm}$ in length and $5 \mathrm{~cm}$ in width used for oropharyngeal reconstruction; the average size was $7.15 \mathrm{~cm}$ long and $3.73 \mathrm{~cm}$ wide. An overview of the clinical series is described in Table 1.

Seven patients had an oral cavity carcinoma. Three were T2 lesions of the floor of the mouth, 1 was a T4a of the left mobile tongue, 1 was a T1 retromolar trigone carcinoma, 1 was a second primary $\mathrm{T} 1$ buccal mucosa carcinoma (in the field treated 7 years before with brachytherapy), and 1 was an alveolar process T4a carcinoma with bony invasion that required a segmental mandibular resection. In this patient, an intraoral soft tissue reconstruction using the IHFMCF was combined with osseous fibula free flap reconstruction. Five patients had oropharyngeal carcinoma: 1 was a T2 unilateral soft palate carcinoma, 1 was a T4a base of tongue (this patient required the widest skin paddle of the series, $9 \times 5 \mathrm{~cm}$, so that a deltopectoral flap was used to close the donor site), while the remaining 3 patients presented with an unilateral $\mathrm{T} 4 \mathrm{a}$ vallecula carcinoma (the tumor extended inferiorly to the epiglottis and homolateral aryepiglottic fold and superiorly to the base of tongue). A partial pharyngolaryngectomy with a controlateral pharyngotomy approach was combined with IHFMCF reconstruction of 


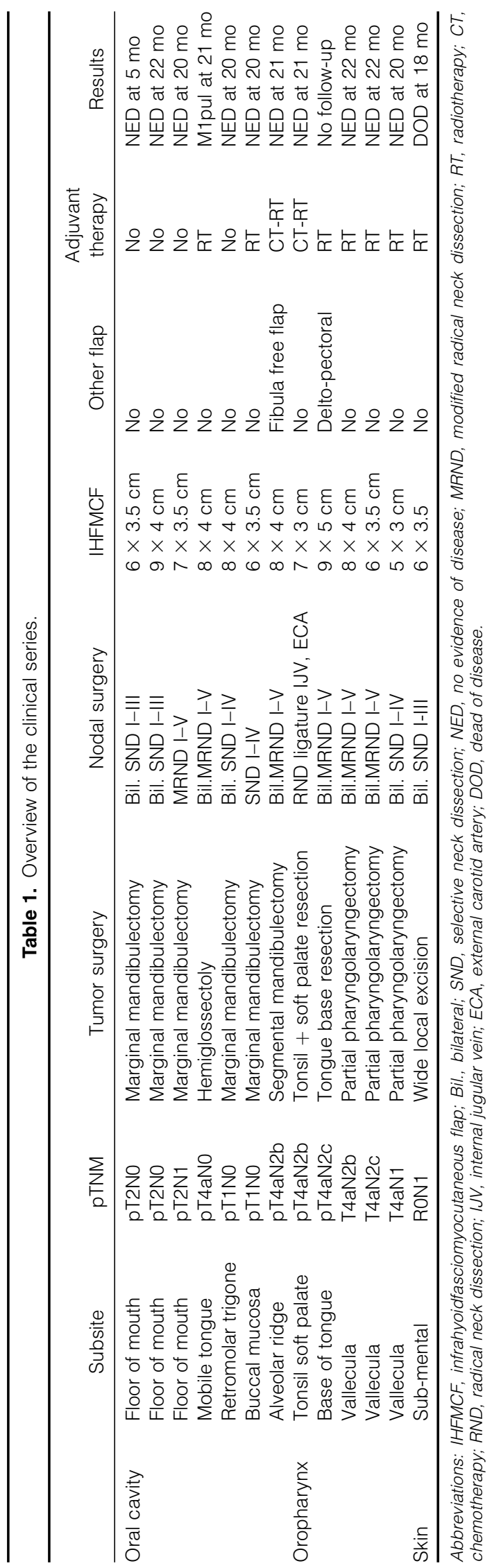

the resected pharyngolaryngeal unit. One patient had a Merkel cell carcinoma of the submental skin region and was treated with wide local excision and bilateral selective neck dissection. ${ }^{8}$

All the flaps were harvested from the same neck side as the primary tumor during homolateral neck dissection; 11 patients had bilateral neck dissection. In 1 case, the internal jugular vein and external carotid artery were ligated above the braching of the superior thyroid pedicle to allow for a safe removal of a lymph node metastasis at level IIa without compromising the vitality of the flap. The reliability of the IHFMCF reconstruction was evaluated in terms of possibility to reach the recipient site, shape matching between the defect and the skin paddle, vitality after transposition, and definitive integration. Postoperative vitality of the flap was checked by clinical observation only. In the series, 9 patients underwent postoperative therapy; 2 patients received concomitant chemoradiation, and the remaining 7 patients received radiation therapy alone. Twelve patients, excluding the patient with Merkel cell carcinoma, required a temporary tracheotomy that was closed within 2 weeks. The nasogastric feeding tube was removed within a week in 10 patients and within 20 days in the 3 patients that underwent partial pharyngolaryngeal resection.

Surgical Technique. The cervical incision is outlined as shown in Figure 1, the skin paddle always being located at the same neck side of the tumor resection. The medial limit of the IHFMCF lies at the midline, the upper limit at the level of the hyoid bone, and the lower limit at the suprasternal notch, the lateral limit lies 3 to $5 \mathrm{~cm}$ from the midline. The shape of the flap is rectangular in a vertical position.

While performing the incision, we suggest immediately incising the skin and platysma all

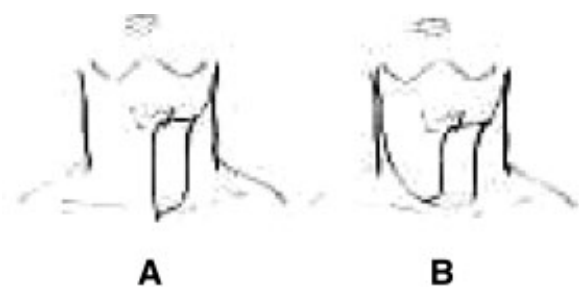

FIGURE 1. (A) Cervical incision if unilateral neck dissection is needed. (B) Cervical incision if bilateral neck dissection is needed. 
around the skin paddle to allow prompt choke perforator vessels opening.

The cervical skin flap are elevated as during a standard neck dissection, the superficial cervical fascia along the anterior border of the sternocleidomastoid muscle, from the sternal insertion up to the level of the hyoid bone, is incised, and the dissection of the fascia proceeds until the intermediate tendon of the omohyoid muscle is identified at its intersection with the internal jugular vein. The tendon is divided and subfascial dissection is carried on toward the flap, suturing this portion of the fascia and the stump of the omohyoid muscle to the lateral edge of the skin paddle (Figure 2).

After modified radical or selective neck dissection is completed (the preservation of the superior thyroid and internal jugular veins is mandatory), the dissection of the flap starts by dividing the anterior jugular vein and sectioning the sternohyoid and sternothyroid muscles distally at the level of the suprasternal notch (Figure 3).

The skin paddle is stitched to the underlying muscles and then the IHFMCF is raised over the avascular plane of the proper capsule of the thyroid gland; when the dissection reaches the upper pole, the cricothyroid artery and vein, all the distal branches of the superior thyroid artery and vein that supply the thyroid gland and the posterior branch of the superior thyroid artery and vein at their entrance in the upper pole of the gland are legated, divided, and kept with the flap (Figure 4).

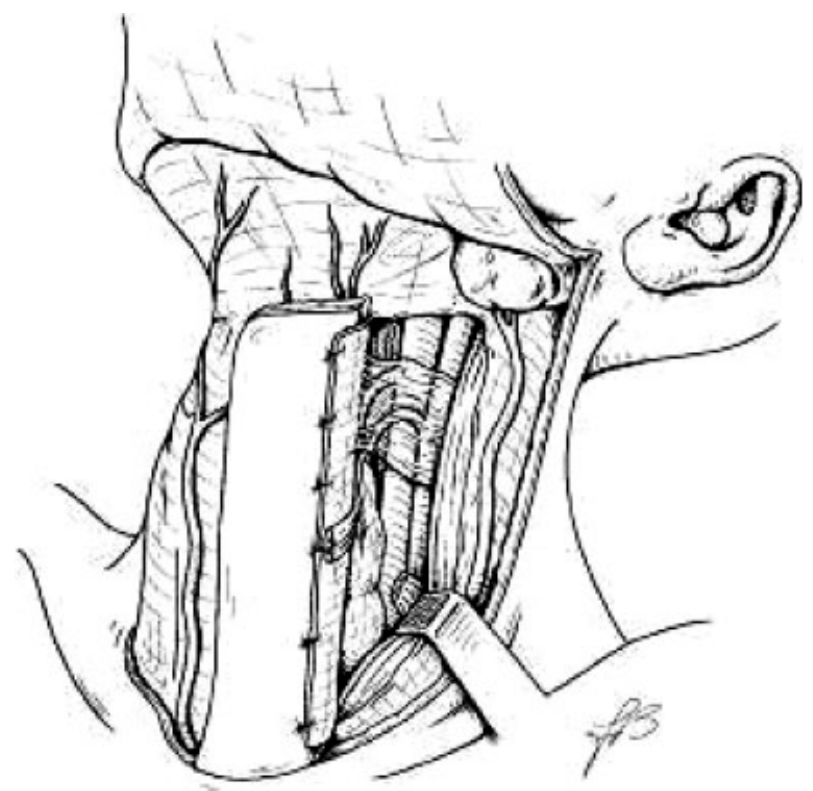

FIGURE 2. The tendon of the omohyoid muscle is divided and subfascial dissection is carried on toward the flap.

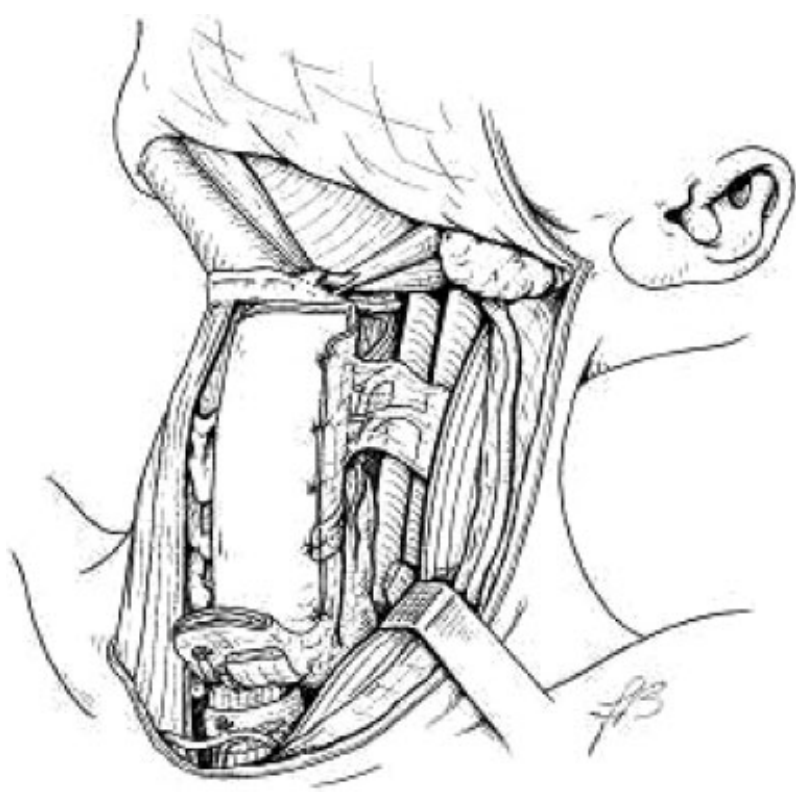

FIGURE 3. The dissection of the flap starts by dividing the anterior jugular vein and sectioning the sternohyoid and sternothyroid muscles distally at the level of the suprasternal notch.

The sternothyroid muscle is detached from the thyroid cartilage.

Special care must be taken in preserving the external branch of the superior laryngeal nerve; therefore, the thyrohyoid muscle is usually spared and left in place. Finally, the hyoid insertion of the

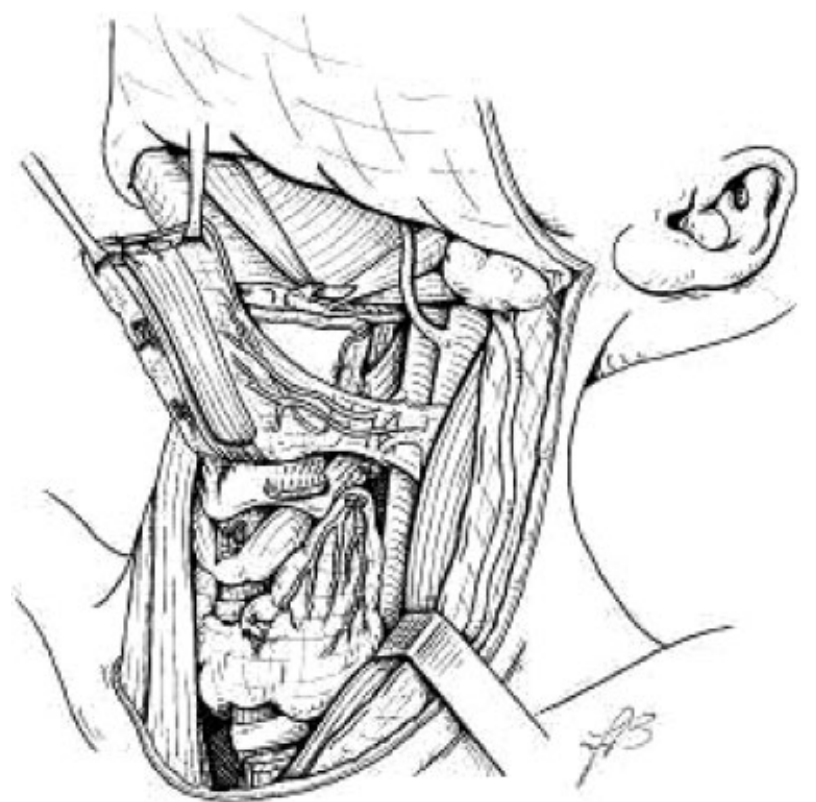

FIGURE 4. When the dissection reaches the upper pole of the thyroid gland, all the distal branches of the superior thyroid artery and vein that supply the thyroid gland are individually legated, divided, and kept with the flap. 


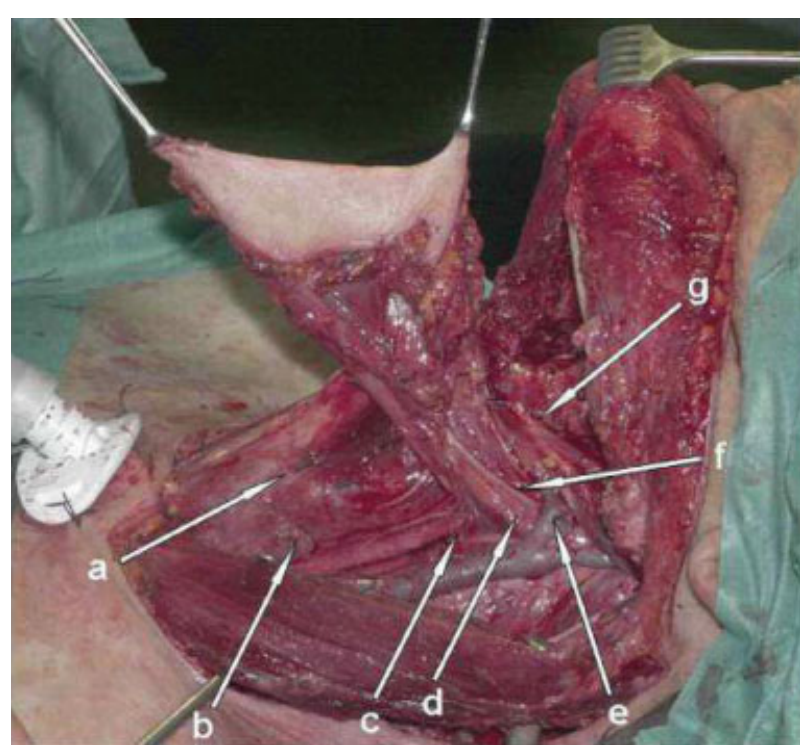

FIGURE 5. a: cricothyroid artery; b: posterior branch of the superior thyroid artery at the entrance in the upper pole of the thyroid gland; c: fascial connections between superficial and median cervical fascia; d: superior thyroid artery; e: superior thyroid vein; f: ansa cervicalis; g: periosteal connections to the digastric muscle. [Color figure can be viewed in the online issue, which is available at www.interscience.wiley.com.]

sternohyoid and omohyoid muscles are sectioned inside out in a subperiostial plane. The pedicle of the flap is formed by the neurovascular pedicle (superior thyroid artery and vein and ansa cervicalis), by fascial connections between the superficial and median cervical fascia, and by periosteal connections to the digastric muscle (Figure 5).

Those facial connections are important to directly provide microvascular venous return toward the median cervical fascia and to protect the superior thyroid vein from twisting or kneeing, so creating the new infrahyoid fascio-myocutaneous flap.

The flap is ready to be transferred to reconstruct the defect.

The donor site can be usually primarily closed, if the width of the skin paddle is greater then $5 \mathrm{~cm}$ a deltopectoral flap could be needed.

\section{RESULTS}

In this series, all the flaps reached the recipient area without extensive vascular pedicle stretching, even in case of soft palate reconstruction. The rectangular shape of the skin paddle matched perfectly with the shape of the resections that resulted mostly oval or rectangular (Figure 6). No total or partial flap necrosis was experienced. All

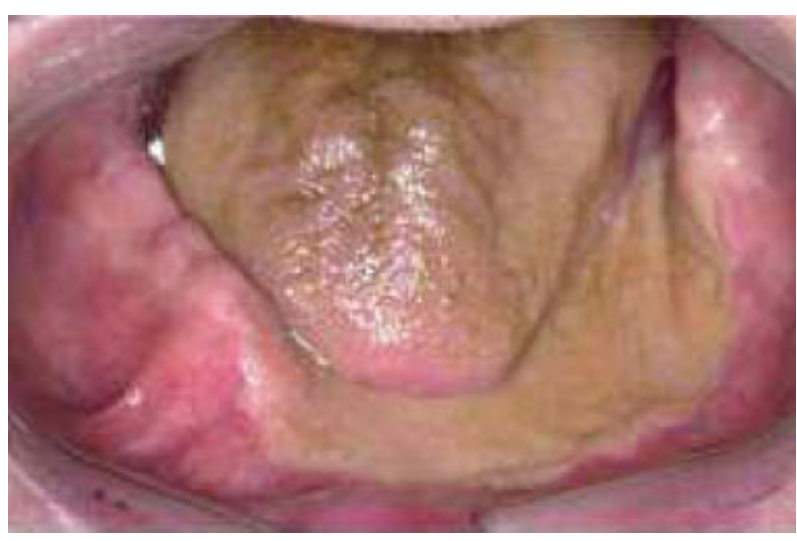

FIGURE 6. Postoperative result after 6 months. The flap covers the marginal mandibulectomy and reconstructs the floor of mouth allowing good tongue mobility. The rectangular shape of the skin paddle matched perfectly with the shape of the resection. [Color figure can be viewed in the online issue, which is available at www.interscience.wiley.com.]

reconstructions healed timely and without wound complications. Also, the healing process at the donor site was excellent in every case, with good aesthetic results, including the patient who needed a deltopectoral flap to achieve donor site closure.

In this series, good functional results were achieved; all patients were decannulated and the nasogastric feeding tube was removed with restoration of oral intake in all cases. In every case, the flap withstood adjuvant treatments without any local complication, and the long-term results as to reconstruction appearance were excellent. We experienced no flap fibrosis, and only 1 patient experienced hair growth in the skin paddle.

\section{DISCUSSION}

In this series, 7 patients of 13 presented with a relative contraindication for a FRFF reconstruction: 2 patients had systemic vascular insufficiency, 1 patient was HIV positive with poor general condition, 1 patient was a professional piano player, and 3 patients were elderly and in poor general condition.

The advantages of the IHFMCF include its easy and relatively quick preparation, and a flap that is harvested during neck dissection so there is no need for a second surgical team. The skin paddle is hairless in most cases, and in almost every case the donor area can be primarily closed avoiding skin grafting or scars beyond the head and neck area, with absence of significant cosmetic and functional squeals. On the other hand, FRFF reconstruction mostly requires 2 surgical teams, an expert microsurgeon, and vigilant mon- 
itoring of the free flap during the first postoperative days.

The majority of myocutaneous flaps for head and neck reconstruction (eg, pectoralis major, trapezius, latissimus dorsi) are quite bulky, and for this reason, we found that the IHFMCF represents an excellent alternative to FRFF reconstruction for medium-sized defects of the oral cavity and oropharynx, which can also easily reach sites such as retromolar trigone and soft palate.

The IHFMCF is thin and pliable, and even if it is not as thin and pliable as the FRFF, it appears to be extremely suitable in case of floor of mouth reconstruction, especially in case of marginal mandibulectomy and en bloc resections, because it is able to provide thigh closure preventing salivary fistulas in the neck and it allows good motility of the tongue.

In 1 case, where a segmental mandibulectomy was performed, we combined the IHFMCF with a free fibula reconstruction. If the mucosal loss is not very large, the IHFMCF suits the defect perfectly and the osseous microvascular transfer is well covered by vascularized infrahyoid muscles.

In case of tongue reconstruction, it is useful to preserve the motor innervation of the infrahyoid muscles provided by the ansa cervicalis. The main advantage of this voluntary innervated flap is that the innervation prevents scarring and atrophy of the reconstructed tongue. ${ }^{6}$ For intermediate oropharyngeal defects, we found that the IHFMCF suits the defect perfectly if the defect does not extend into the oral cavity. On the other hand, if a pharyngeal defect does extend to the oral cavity, a complex reconstruction in terms of dimensions and shape is needed, and the FRFF appears to be preferable. For soft palate reconstruction, if the resection is strictly uniolateral and does not include the uvula, the IHFMCF can be used with good functional results preventing open rhinolalia and providing soft palate competence without nasal regurgitation. If a larger soft palate defect exists, a double-folded FRFF is functionally superior. In case of partial pharyngolaryngectomy for vallecula carcinoma, the IHFMCF provided an excellent restoration of form and function, being small and pliable.

All flaps in this series were harvested with the inclusion of the fascia cervicalis superficialis, which assists microvascular tissue drainage toward the median cervical fascia. For this reason, it is important to keep a certain amount of fascia around the superior thyroid pedicle without thinning it too much during the dissection. This maneuver does not interfere with the oncologic radicality of the neck dissection.

\section{CONCLUSIONS}

A critical recodification of the role of the infrahyoid flap in modern days could be of great help for the microvascular surgeon looking for alternatives, because in management of head and neck tumors the tool box of a wide range of reconstructive options is of a great advantage. In our series, the IHFMCF has shown to be a reliable flap even in elderly patients and in patients in general poor condition or with peripheral vessel insufficiency who are not optimal candidates for free flap reconstruction. The use of the described technical modifications together with the inclusion of part of the superficial cervical fascia in the harvest has led to a complete success rate without venous problems in this series.

This flap is thin and pliable, so that it is particularly useful in oral cavity reconstructions. In this preliminary experience, our impression is that for small and medium-sized defects the functional results are comparable to those with the FRFF reconstruction. FRFF appears to be preferable for the reconstruction of extensive oropharyngeal defects where a large amount of skin is needed, but for the closure of small and mediumsized defects and after partial pharyngolaryngectomy, IHFMCF has proved to be an excellent alternative.

Contraindications such as previous neck dissection, previous thyroid surgery, and presence of N3 neck disease must be respected. A relative contraindication is previous radiotherapy. If it is possible to preserve the superior thyroid pedicle dividing the internal jugular vein and external carotid artery just above its branching, the infrahyoid flap can be harvested also if metastatic lymph nodes are present at level II. In our series, 8 patients presented a $\mathrm{pN}+$ neck at the side of the flap, and it was always possible to use the planned IHFMCF despite the proximity to a metastatic node.

Acknowledgment. The authors thank Francesca Brunone for the surgical diagrams.

\section{REFERENCES}

1. Evans GR, Schusterman MA, Kroll SS, Miller MJ, et al. The radial forearm flap in head and neck reconstruction: a review. Am J Surg 1994;168:446-50.

2. Wang HS, Shen JW. Preliminary report on a new approach to the reconstruction of the tongue. Acta Acad Med Prim Hanghai 1980;7:256-259.

3. Magrin J, Kowalski LP, DiPaula RA, et al. Infrahyoid myocutaneous flap in head and neck reconstruction. Head Neck 1993;15:522-525. 
4. Wang H, Junwen S, Tian A, et al. The infrahyoid myocutaneous flap for reconstruction after resection of head and neck cancer. Cancer 1986;57:663-668.

5. Rojanin S, Suphaphongs N, Ballantyne AJ. The infrahyoid musculocutaneous flap in head and neck reconstruction. Am J Surg 1991;162:400-403.

6. Remmert SM, Sommer KD, Majocco AM, Weerda HG. The neurovascular infrahyoid flap: a new method for tongue reconstruction. Plast Reconstr Surg 1997;99:613618.

7. Zhao YF, Zhang WF, Zhao JH. Reconstruction of intraoral defects after cancer surgery using cervical pedicle flaps. J Oral Maxillofac Surg 2001;59:1142-1146.
8. Deganello A, De Bree R, Dolivet G, Leemans CR. Infrahyoid myocutaneous flap reconstruction after wide local excision of a Merkel cell carcinoma. Acta Otorhinolaryngol Ital 2005;25:50-54.

9. Wang H. 10 years' experience on infrahyoid myocutaneous flap. Zhonghua Er Bi Yan Hou Ke Za Zhi 1991;26: $332-334,382$.

10. Dolivet G, Gangloff P, Sarini J, Ton Van J, Garron X, Guillemin F, Lefebvre JL. Modification of the infra hyoid musculo-cutaneous flap. Eur J Surg Oncol 2005;31:294-298.

11. Sobin LH, Wittekind Ch, editors. TNM classification of malignant tumors, 6th ed. International Union Against Cancer; New York: Wiley-Liss; 2002. 